\title{
EL PROCESO DE INICIACIÓN AL CURANDERISMO EN LA PAMPA (ARGENTINA)
}

\author{
INITIATION PROCESS IN TRADITIONAL HEALER IN \\ LA PAMPA (ARGENTINA)
}

\author{
Facundo Arteaga ${ }^{1}$
}

\begin{abstract}
El propósito de este artículo es mostrar, mediante una profunda descripción analítica, los complejos procesos de iniciación y obtención de poder por parte de los curanderos entre los criollos de la provincia de La Pampa, Argentina. Sobre la base de materiales etnográficos originales se describen y analizan detalladamente los conocimientos acerca de remedios vernáculos y técnicas terapéutico-rituales, así como el conjunto de actividades que denotan las prácticas curanderiles como resultado de la iniciación y que van más allá de la terapia, tales como el daño y la adivinación. En segunda instancia, se presentan y examinan los procesos de iniciación propiamente dichos y los caminos de obtención de poder, señalando la existencia de dos alternativas principales: (a) el traspaso de poder y conocimiento de parte de otro curandero y (b) la superación de una experiencia vital de carácter crítico, considerando especialmente la adquisición de auxiliares (vírgenes, santos y muertos).
\end{abstract}

Palabras claves: medicina tradicional, curandero, La Pampa, Argentina.

The aim of this article is to show, through deep analytical description, the complex processes of initiation and empowerment of traditional healers among European Argentines - mostly Spanish and Italian in origin-who settled in the Province of La Pampa, Argentina. Based on original ethnographic research, I describe and analyze knowledge about traditional remedies and therapeuticritual techniques, as well as the practices of traditional healers as a result of initiation, such as damage and divination. Secondly, the proper initiation processes and ways of obtaining power are presented and discussed, distinguishing two main alternatives: (a) the transfer of power and knowledge from one healer to another member of the community prior to initiation and $(b)$ overcoming a life experience of a critical nature, especially considering the procurement of helpers (virgins, saints and dead people).

Key words: Traditional medicine, folk healer, La Pampa, Argentina.

La práctica curanderil es una de las dos modalidades terapéuticas de la medicina tradicional en Argentina ${ }^{1}$, la otra es el shamanismo practicado en las sociedades indígenas del Gran Chaco, la selva Subtropical misionera y la Patagonia.

El curanderismo fue traído primero por los conquistadores españoles y más tarde por los inmigrantes europeos, entre los que se destaca el impacto poblacional de italianos y españoles. Las prácticas de estos especialistas, llamados curanderos o médicos de campo, si bien admiten variaciones de individuo a individuo, poseen un gran número de elementos comunes que permiten identificarlos y distinguirlos de otro tipo de especialistas.

En lo que respecta a Argentina, es la medicina tradicional más difundida, presente tanto en áreas rurales como urbanas. Sus concepciones y tratamientos como ha notado Idoyaga Molina (2000) sintetizan saberes populares, antiguas praxis y conocimientos de la medina occidental, muchos de ellos de raigambre humoral, con terapéuticas rituales de origen católico en su mayoría. En el caso particular de La Pampa, dicha síntesis se puede observar a través de: (a) antiguas concepciones de la medicina académica, devenidas populares en muchos casos antes de que los inmigrantes se instalaran en el área de estudio, (b) tradiciones populares propias de las medicinas tradicionales española e italiana y (c) terapias rituales en su mayoría de raigambre católica o asociadas a elementos del catolicismo, muchas de las cuales fueron praxis de las elites y luego devinieron en prácticas populares, tales como la cura por medio de ensalmos, el sahumado y los rezos. Sin embargo, no podemos obviar que en lo que a síntesis se refiere, durante la edad media, las tradiciones médicas de los pueblos peninsulares

\footnotetext{
1 Centro Argentino de Etnología Americana/Consejo Nacional de Investigaciones Científicas y Técnicas-Instituto Universitario Nacional de Arte. Buenos Aires, Argentina. facundoarteaga@ gmail.com
} 
sufrieron el impacto de la medicina árabe, sobre todo en su versión humoral. Estos especialistas suelen poseer un acabado conocimiento de las funciones terapéuticas de vegetales, de ciertos minerales y de elementos animales -como grasas y la piedra bezoar de los camélidos-que les permiten administrar a sus pacientes los remedios que consideren adecuados.

Al hablar del saber popular de los inmigrantes, nos referimos a las tradiciones aportadas por los colonos que se asentaron en este territorio entre fines del siglo XIX y principios del XX, después de que la llamada Campaña al Desierto expulsara a los indígenas Mapuches ${ }^{2}$ del área pampeana. En nuestra zona de estudio se localizaron mayoritariamente españoles -vascos y gallegos en su mayoría-e italianos y en segundo término se destacan los sirio-libaneses a los que se sumaron asentamientos alemanes ya avanzado el siglo XX. Estos saberes y prácticas se advierten en el reconocimiento, diagnóstico y tratamiento de taxa vernáculos como el mal de ojo, empacho, culebrilla, pata de cabra, brujería o mal hecho, desgarros, entre muchos otros ${ }^{3}$, muchos de los cuales fueron antiguamente saberes de elite.

La terapéutica ritual incluye rezos, invocaciones, pedidos y encendido de velas a las deidades cristianas, la utilización y consumo de agua bendita, la triple repetición de acciones que implican la manipulación del poder del tres -número sacralizado por su asociación a la Trinidad-, el uso de agua y aceite, el sahumado de los pacientes y de los espacios corrompidos, la curación por ensalmos y la ejecución de la señal de la cruz, símbolo de vida y restauración, como han notado en relación con otras regiones del país Idoyaga Molina (2001, 2002). Estas concepciones del catolicismo también aportaron los saberes actuales sobre las prácticas brujeriles, almas en pena, rituales en celebración de los muertos, festejos de carnaval y otros hechos que van cayendo o han caído en desuso en Europa. El folclore europeo también está presente en creencias sobre augurios y presagios de índole nefasta y positiva, sobre seres míticos como el lobisón o sobre pactos con el diablo, que de uno u otro modo se ligan al ámbito de la enfermedad y la muerte.

Los curanderos no deben pensarse como portadores de conocimientos cristalizados en el tiempo, sino como especialistas que incorporan y refiguran constantemente diversos elementos, tipos de servicios y saberes que les permiten adaptarse al nuevo contexto local y global, convirtiéndose en una opción apreciada por individuos de distintos sectores socioeconómicos y educacionales. La dinámica de cambio se advierte muy fácilmente en su práctica cotidiana cuando recomiendan a sus pacientes tratarse no sólo con remedios tradicionales sino también con fármacos de laboratorio, combinación que se ha vuelto típica en las sugerencias de estos especialistas, como se ha señalado en relación con otras áreas (Idoyaga Molina 2002).

El curandero practica una terapia holística en la que es imposible separar sus conocimientos sobre la preparación y uso de remedios tradicionales ${ }^{4}$ de su capacidad para manipular lo sagrado en el contexto de acciones terapéuticas rituales, por lo cual es sumamente interesante conocer su proceso de iniciación y de aprendizaje, proceso que lo transformará finalmente en el especialista de esta medicina. Es por eso que esta medicina no debe escindirse en dos dominios, como lo proponen algunos investigadores (1978; Pérez de Nucci 1988), el de un sab Palma er empírico verificable por la ciencia, producto del ensayo y el error y otro componente místico, mítico o sobrenatural que hace a la manipulación de lo sagrado en la terapéutica ritual; de hecho la preparación de los remedios no se separa del ritual, así los elementos adosados a una cataplasma se rocían dibujando una cruz, o la infusión de tal o cual vegetal se prepara en agua bendita.

Si bien otros autores han trabajado esta temática en diferentes zonas del país (Bianchetti 1994; Colatarci 1999; Disderi 2001; García 1984; Idoyaga Molina 1997, 1999, 2001, 2002; Kalinsky y Arrué 1996; Palma 1978; Pérez de Nucci 1989; Ratier 1972; Sturzenegger 1987; Torres y Aprea 1995), en la región de estudio el material es aún incipiente y no se ha tenido en cuenta la perspectiva de los actores.

En esta oportunidad intentamos contribuir al conocimiento del curanderismo en La Pampa (Argentina), especialmente enfocamos el proceso de iniciación analizando las formas de adquisición de poder, de auxiliares y de saberes específicos que le permitirán al nuevo curandero ejercer las actividades que lo distinguen.

El material sobre el que nos basamos fue recogido personalmente en varias campañas, realizadas entre los años 1999 y 2006 en las que utilizamos técnicas cualitativas. La información de mayor importancia para este trabajo fue recabada a través de entrevistas abiertas, extensas y recurrentes con informantes calificados, y/o grupos naturales (Coreil 1995). Respecto de los tópicos abordados consideramos: el llamado a la actividad 
de curandero, las variantes existentes (si es que las conocen), la adquisición de poder -en sentido de sagrado- y de los auxiliares, el conocimiento de los taxa y sus correspondientes etiologías y tratamientos, entre otros. Se procedió también a la observación y a la observación participante de diferentes praxis terapéuticas llevadas a cabo por curanderos. Para el análisis de los datos se procedió a la utilización de un enfoque hermenéutico y fenomenológico. Respecto de los materiales originales, diremos que las entrevistas se dividieron en diferentes grupos, de acuerdo al rol de los entrevistados (curanderos, legos, pacientes, familiares de pacientes y trabajadores sociales). Cada uno de los grupos fue analizado en función de los siguientes ejes:

(a) Taxa vernáculos de enfermedades: etiologíadiagnóstico y terapia.

(b) Caminos-trayectorias para acceder al estatus de especialista, la adquisición de auxiliares en caso de existir, la capacidad de curar y otras actividades, como los procesos de daño y contradaño.

La labor de campo se llevó a cabo entre la población rural de los departamentos de Utracán, Limay Mahuida, Toay, Curacó, Lihuel Calel y Capital de la provincia de La Pampa; se trata de una población de tres a cuatro generaciones en la zona, básicamente ligada a las tareas agrícolasganaderas. Consideramos informantes calificados a: curanderos, enfermos, familiares de enfermos y legos practicantes de la medicina doméstica o autotratamiento. En lo que hace a los terapeutas tradicionales enfocamos el proceso de iniciación.

El trabajo con curanderos nos permitió detectar las adaptaciones que los mismos realizan en función de las nuevas demandas, resultantes de los cambios y la multiplicación de los perfiles de los consultantes.

El enfoque metodológico propuesto apunta a concretar una etnografía que dé cuenta de las vivencias y significados que implican las prácticas del curanderismo para los actores sociales, tanto los especialistas como los usuarios, enmarcándose en la hermenéutica fenomenológica que apunta a la comprensión de los fenómenos culturales en toda su amplitud y complejidad, en cuanto contenidos de conciencia intersubjetivos. Para lo cual en primera instancia es necesario suspender o poner entre paréntesis los saberes teóricos y tradicionales, así como las disposiciones pragmáticas y afectivas -que distorsionarían el proceso cognitivo que transforma el hecho vivido en dato del investigador- (Merleau Ponty 1945), para acceder a las vivencias y experiencias del sujeto, las que se constituyen en el núcleo central del análisis. En un segundo momento, se procedió a la concreción de un acabado fenomenismo que brindó todas las facetas del hecho analizado, en nuestro caso el curanderismo. Es así que consideramos al hecho o fenómeno cultural como una estructura que sólo puede ser comprendida teniendo en cuenta todas las asociaciones y significados para que devele el hecho como contenido de conciencia. Esto es, en tanto hecho pensado, actuado y vivido.

\section{El Proceso de Iniciación}

El curandero es un terapeuta tradicional que trata los diferentes tipos de desequilibrios que trae aparejada la enfermedad ${ }^{5}$. Es reconocido como tal por la población, quien lo señala como especialista para la atención de diferentes males. Es a su vez señalado y caracterizado como un personaje ambivalente, porque no solamente ejerce la terapia sino que además es capaz de realizar "trabajos de daño"6. Este tipo de acciones implican el uso o manipulación de lo sagrado con una intencionalidad negativa, que se dirige a dañar a un individuo, su familia o sus pertenencias -los actores sociales señalan como objetos preferenciales del daño a casas, vehículos, cosechas o animales en general-. Pero para acceder al estatus de curandero tiene que atravesar una suerte de iniciación en la que adquiere poder, auxiliares y conocimientos.

En Argentina diversos autores han señalado distintos modos de iniciación entre los que aparecen el nacimiento con el poder innato, la herencia, la cesión de las dotes por otro curandero, sobrevivir después de ser atacado por un rayo, superar estados de enfermedad o experiencias críticas en la vida del individuo, ser hermano mellizo o deforme, la aparición de un ser otro en sueños -ya sea un santo u otro-, entre otras posibilidades (Colatarci 1999; Di Lullo 2005 [1947]; Idoyaga Molina 1997; Palma 1978; Pérez de Nucci 19833; Ratier 1972; Sturzenegger 1994), los que básicamente detallan similares características en relación con el curanderismo en España (Briones Gómez 1996; Gómez García 1996; Mariño Ferro 1996). 
En nuestro trabajo de campo -como desarrollaremos más adelante- encontramos dos vías principales para alcanzar el estatus de curandero:

(a) Enseñanza de un curandero a un aprendiz.

(b) El llamado a la actividad a partir de:

(b.1) Sortear una situación de experiencia crítica de vida (como por ejemplo un accidente o enfermedad).

(b.2) El contacto de una deidad u otro ser con características de hierofanía ${ }^{7}$ (Eliade 1964).

Estas formas de iniciación mencionadas sólo difieren en las maneras de acceder al poder y al conocimiento, pero no así en las capacidades obtenidas como terapeutas.

Respecto a los antecedentes en la campaña argentina de estos terapeutas, ya en el año 1908 Boman en su paso por la Puna relata su encuentro con una "médica/curandera" que atiende a uno de los peones que lo acompañaban en su expedición por propio pedido de este último. Más adelante, en 1947, Di Lullo sostiene que la curandera es una persona que cumple una gran función social en el campo santiagueño. En este sentido, Ratier (1972) plantea que estos especialistas tienen un llamamiento o iniciación a esta actividad a través de diferentes modos, como por ejemplo ser alcanzado por un rayo, el cual no sólo le da el estatus y el poder para las diferentes actividades que realizará, sino que además pareciera transmitirle los conocimientos acerca de las plantas con cualidades curativas. Palma (1978) concibe dos formas mediante las cuales el curandero adquiere su capacidad médica en el Noroeste Argentino: (a) a través de la enseñanza de un curandero, proceso en el cual aprenderá a reconocer las plantas medicinales, sus propiedades y la forma de administración, incorporando saberes acerca de diferentes etiologías y sintomatologías. En algunos casos la transmisión de conocimientos se realiza de padres a hijos, aunque también se puede hacer entre personas con cierto grado de parentesco o simplemente conocidos. (b) por medio de una singular circunstancia como cuando una persona es alcanzada por un rayo sufre un proceso de desintegración y de re-integración, pero para que este último resucite no tiene que haber testigos del suceso. Este hecho es sindicado como un señalamiento de Dios para ser curandero. La diferencia de un iniciado a otro según el autor, es que el primero podrá atender las enfermedades en las cuales se instruyó, mientras que el segundo podrá atender todos los casos que le lleguen.

Creemos que esta afirmación no es completamente acertada; por un lado, porque dentro del proceso de iniciación de los curanderos está contemplado el entrenamiento, y distinguirlos de esta manera llevaría a confundir a un curandero con un lego.

En esta gran zona, más específicamente en los Valles Calchaquí, Pérez de Nucci (1988) afirma que el curandero presenta rasgos definidos y característicos que pueden identificarlo socialmente como tal, pudiendo asumir este rol por distintos caminos. Uno es lo que denomina por predestinación, en cuyo caso la fuerza mágica le es dada desde su nacimiento, lo que está íntimamente ligado a la posibilidad de que dicha predestinación le sea conferida como herencia por alguno de sus progenitores. La segunda posibilidad es cuando al curador el poder le es dado en un acontecimiento crucial de su existencia, como una enfermedad o accidente de gran magnitud que pone en peligro la vida del iniciado -aquí ubica a las personas que fueron alcanzadas por un rayo, quienes de acuerdo con los actores se desintegran y vuelven a la vida convertidos en curanderos-. La tercera forma que describe es la del curador sucesorio, el cual recibe de un curandero consagrado que está en la última etapa de su vida los conocimientos y el poder para curar, efectuándose mayoritariamente de padres a hijos. A este proceso se le sumará el aprendizaje de los conceptos etiológicos y sintomatológicos de las afecciones más comunes, la sabiduría para identificar las plantas y sus propiedades junto con la distinción de los elementos minerales y animales que son útiles para el diagnóstico y cura de las enfermedades.

Dentro de las formas de iniciación descritas por el autor, no podemos dejar de notar -como lo adelantáramos-, que considerar la idea de "fuerza mágica" recibida al nacer es seguir pensando la dualidad antiguamente planteada de la medicina tradicional: saber empírico verificable por la ciencia más el componente mágico-supernatural.

Por otra parte, algunos autores al referirse a la zona de la provincia de Neuquén (Kalinsky y Arrué 1996) sostienen que las curadoras populares de esa provincia, al igual que las machis mapuches, tienen que sortear una iniciación en la cual existe una revelación para transformarse en sanadora. Sin 
embargo, la iniciación es un proceso que sortean todos los shamanes y una gran parte de los curanderos, dato último que surge de diversas relevaciones en Argentina.

Continuando con la revisión y análisis acerca de los procesos de iniciación en diferentes partes del territorio argentino, Sturzenegger (1987), quien desarrollara su trabajo de campo en Formosa, nota que sólo se reconoce como curadores de la medicina criolla a quienes tienen la capacidad de diagnosticar y de tratar un número elevado de enfermedades, tratándose de individuos que casi sin excepción se consagran exclusivamente en el arte de curar. Este aprendizaje de la medicina tradicional comienza en su infancia del mismo modo que aquellos que no se convierten en curadores en el proceso general de socialización del niño. En algunos casos hay una voluntad por parte de un adulto en vista a formar a un niño particular generalmente miembro de esa familia, pero no es sino más tarde que se inicia un acontecimiento consagratorio. Puede tener lugar a través de la transferencia al joven del poder del curador el cual abandona sus prácticas, un sueño revelador o una experiencia meta-sensible lo que imprime definitivamente en el individuo su nueva condición (Sturzenegger 1987).

La descripción de la autora nombra tres formas de iniciación al igual que casi todos los autores antes mencionados, sin embargo, no describe claramente dicho proceso. Tampoco define lo que considera como experiencia meta-sensible, lo que nos deja con poco corpus para comparar con nuestra zona de estudio.

Briones Gómez (1996), para España, plantea que para ejercer el oficio del curandero con la aprobación social hará falta que el individuo pase por un proceso que lo transforme, lo capacite y le dé los poderes y la autoridad que legitiman en el interior de una sociedad y cultura dadas. En palabras del autor, refiere que se nace curandero y no se hace, se accede desde este estatus por nacer con el don de curar, que luego se revela a través de una instancia exterior no natural, lo que denomina una innovación carismática. Pero también refiere que muchas veces los poderes son traspasados entre familiares, de abuelas a nietas o de padres y madres a hijos.

El gran trabajo del autor para la provincia de Granada en España encuentra muchos puntos comunes con el proceso de iniciación en el curanderismo de La Pampa. Sin embargo, se observan ciertas diferencias, principalmente, en lo que concierne a la afirmación de que "curandero se nace y no se hace". Esta idea no se encuentra entre los datos relevados en nuestro trabajo de campo, como lo demostraremos a continuación.

En la provincia de La Pampa -como adelantáramos al inicio de nuestro trabajo- podemos observar diferentes tipos de inicia ión, pueden remitirse desde la cesión de poder por parte de otro curandero, la revelación de la capacidad después de vivir enfermedades o experiencias trágicas de vida, o bien por la revelación de una deidad, lo que se interpretaría como un llamado a la actividad. Con la revelación, en algunos casos, se adquieren también saberes sobre el uso de etnofármacos, cataplasmas, ventosas y otras herramientas terapéuticas. Sin embargo, en otros casos, estos saberes pueden ser adquiridos a lo largo de la vida, es decir, que el iniciado ya lo aprendió antes de tener poder.

En el fragmento del siguiente relato podemos observar una de las vías de iniciación. El proceso de aprendizaje, en este caso, surge a partir de que una curandera, de edad avanzada y enferma, decide pasarle sus conocimientos y parte de su poder a una persona que concurría a sus terapias:

Ella al tenerle yo tanta fe, un día yo fui a verla y Doña Petrona, como puedo hacer si usted, vos mi 'ja lo vas a curar", yo es como el médico que te diga a vos vos vas a curarte, yo le dije no Doña Petrona, yo no, si míja, porque me tenés mucha fe, y un poquitito de mi poder que ahora que estoy enferma te lo puedo dar...Yo invoco el nombre de ella, y al Espíritu Santo más vale. (Angela, 63 años de edad y 37 años como curandera. Zona de Limay Mahuida). A mí me enseñó una vecina de la vuelta de mi casa que curaba y yo iba siempre a hacerme atender. Me enseño las palabras, las técnicas para curar y me dio el poder para poder ser curandera porque ella estaba viejita ya. (Norma, 44 años de edad y 16 años como curandera. Zona de Toay).

En ambos relatos se observa cómo una curandera anciana decide ceder parte de su poder a una mujer adulta que especialmente respetaba a la anciana dando comienzo así al proceso de iniciación. En este proceso la vieja curandera cede parte de sus poderes y cuando muera pasa a ser una auxiliar de la reciente iniciada. En cuanto a las vivencias de 
ambas especialistas iniciadas, en un primer momento significó cierto temor no sólo a la práctica en sí misma sino a las controversias que vivían dentro de la sociedad por ser curanderas. Pero, por otra parte, sintieron un gran compromiso con la posibilidad de contribuir a su comunidad como lo habían hecho sus predecesoras, devolviendo la salud y ayudando como ellas habían sido ayudadas. Cabe mencionar también, que el proceso de iniciación involucró el conocimiento de ensalmos o fórmulas para curar de palabra ${ }^{8}$. Este aprendizaje, a diferencia del que realizan los legos - del que dan cuenta diversos autores (Bianchetti 1994; Disderi 2001; Idoyaga Molina 2001, entre otros)- no se concretó en días especiales, tales como las vísperas de Navidad y de San Juan o en Viernes Santo, sino que, por el contrario, fue hecho diariamente en forma progresiva y sucesiva, con la clara intención de completar la formación del iniciado. Vemos claramente la distinción aquí con lo recogido por Briones Gómez (1996), ya que entre los datos que arrojó de nuestro trabajo de campo, una persona puede iniciarse en la práctica curanderil sin entrar en juego la predestinación al nacer o el destino.

Por otro lado, el segundo tipo de iniciación recogido es justamente haber sorteado una situación crítica de vida:

Esto no se aprende, viene solo. Yo tenía la tienda y yo no creía ni en el empacho viste, y venían la gente y me decía: Estoy descompuesta ¿no sabes medir vos? Los medía y se componían, yo me descomponía pero ellos se componían. Y después del accidente, eso fue como una cosa, como un aviso, porque de ahí, a partir de ahí la gente empezó a ir a medirse, y no sé por qué, pero los componía. Y te digo que no viene gente sólo de acá, vienen de todos lados, me mandan cartas, que tienen tal y cual cosa, y se componen. Incluso vienen médicos, me traen los chicos para que los mida. Mi abuela me enseñó, ella lo traía de su madre, y ella me enseñó cómo se curaba tal cosa, cómo se curaba lo otro. (Yolanda, 52 años de edad y 18 años como curandera. Zona de Utracán).

Después de tanto enferma salí de la cama que estaba postrada y empecé a curar, yo, que nadie pensaba que me iba a levantar terminé curando a la gente, sin decir nada, una vez que se enteraron que me había curado vinieron, primero los conocidos y después ya gente que no conocía. (Mónica, 36 años de edad y 5 años como curandera. Zona Capital).

Se puede observar en los relatos que la iniciación deviene como consecuencia de una situación crítica, sobre una base de poder que ya la distinguía; hecho repetido no sólo en occidente sino en las instituciones shamánicas de los más diversos grupos étnicos ${ }^{9}$. Es a partir de ese momento especial que la persona adquiere su capacidad de curar. También es relevante en este caso destacar que las personas concurren a ellas con fines terapéuticos sin ningún tipo de aviso previo ${ }^{10}$, lo que deja en claro la legitimidad que la sanadora posee frente al grupo social en el que actúa.

Al igual que las primeras curanderas, para ejercer la terapia recurren a diferentes santos católicos, quienes se desempeñan como sus auxiliares y, según algunos relatos de estas especialistas, juegan el rol de intermediarios entre ella y el Dios cristiano, fundamentando tal actitud en que "uno es demasiado pecador" para pedir directamente a la deidad. Según lo expuesto, la sanadora que sufrió el accidente recibió también un entrenamiento de su abuela en saberes prácticos, tales como la preparación de remedios sobre la base de vegetales, productos animales y otras técnicas, así como conocimientos para descubrir las etiologías de enfermedades.

La última forma de iniciación recogida en nuestra labor de campo refiere al llamado de un ser otro o deidad a la actividad de curar.

Una mujer de Regina (provincia de Río Negro) que venía a curar a Acha me dijo: Dios te va a llamar, y bueno habían un montón de cosas me venían pasando... Una noche termino de orar, me acuesto, y me tapo y escucho una voz, una voz rara pero linda, rara que me inculcó miedo, porque no te voy a decir que era fea porque es mentira: "Anahi" AH! Me re asusté y me tapé, bueno, no sé bien cuanto tiempo pasó y de nuevo, yo estaba re despierta, "Anahî", yo me dije qué pasa acá y me tapo, cuando me tapo bien me acuerdo de Samuel, Dios a Samuel lo llamó 3 (tres) veces, entonces me acordé en ese momento... y le dije: si eres tú el que me está llamando vuélveme 
a llamar. Prepárame que Yo voy a trabajar para ti, dame fuerzas y valor. Me volví a tapar y al ratito "Anahí", bueno el señor te ha llamado. (Anahí, 47 años de edad 7 años como curandera. Zona de Curacó).

En este fragmento observamos como el llamado proviene de Dios, y es interpretado como tal para empezar a ejercer como sanadora. Sus auxiliares son, al igual que todos los casos citados, vírgenes y santos católicos, a los cuales acude al momento de la praxis. Los conocimientos diagnóstico-terapéuticos fueron revelados al momento del llamado y posterior aceptación.

Finalmente, debemos notar que ninguno de los curanderos a los cuales tuvimos acceso nacieron con el don o la capacidad innata de curar, por el contrario, ellos en su totalidad accedieron al estatus de curandero por las vías antes descritas.

\section{Conclusiones}

Cabe señalar que otros autores han tratado esta temática en otras áreas del país con diferente grado de profundidad (Bianchetti 1994; Boman 1908; Di Lullo 2005 [1947]; Idoyaga Molina 1999; Palma 1978; Pérez de Nucci 1989; Rarier 1972, entre otros) y han notado que el poder para curar que tienen los especialistas tradicionales puede ser innato y adquirido.

En nuestra investigación en La Pampa (Argentina) podemos afirmar que los procesos de iniciación pueden originarse a partir de la enseñanza de un curandero a un aprendiz o el llamado a la actividad. Desde esta última posibilidad podemos diferenciar dos vías, sortear una situación de experiencia crítica de vida (como por ejemplo un accidente o enfermedad) o el contacto de una deidad u otro ser con características de hierofanía (Eliade 1964).

Por otro lado, se sabe que los curanderos recurren a auxiliares en la praxis cotidiana. Esta utilización se limita a la invocación de diferentes seres poderosos a los cuales se les solicita su intervención para llevar adelante terapias, adivinaciones $u$ otras actividades. Estos auxiliares pueden ser santos, vírgenes o muertos. Los modos de invocación varían de acuerdo a los auxiliares y a los curanderos, conviven la aparición en sueños con la comunicación en estado de vela. Pudiendo además las figuras míticas presentarse al curador sin que su concurrencia fuera solicitada.

A su vez, una de las informantes (Yolanda) nos explicaba que en el momento de la terapia, dirige sus pedidos a diferentes santos y vírgenes, con el fin de solicitar la intervención de los mismos como mediadores ante Dios. Como dijimos, los pecados son un impedimento al trato directo con la deidad y los santos y vírgenes, en su calidad de auxiliares, intermedian, imploran y obtienen el favor de la deidad.

Finalmente, debemos dejar de manifiesto que no consideramos aquí la capacidad de innato porque ninguno de los informantes y curanderos que entrevistamos llegó al estatus especialista por ese camino. Además, las formas de iniciación mencionadas sólo difieren en las formas de acceder al poder y al conocimiento, pero no así en las capacidades obtenidas como terapeutas.

\section{Referencias Citadas}

Arteaga, F. 2006. Procesos de daño y contradaño en La Pampa (Argentina). Scripta Ethnologica XXVIII:79-94.

Barrios, W. 2000. La enfermedad como daño intencional entre los campesinos de Catamarca. Mitológicas XV:37-48.

Bianchetti, M.C. 1994. Daño, ojeo y brujería en el Valle Calchaquí. Actas de las Terceras Jornadas Nacionales de Folklore. Jornadas Nacionales de Folklore III:65-88.

Boman, E. 1992. Antigüedades de la Región Andina. Editorial de la U.N. de Jujuy, San Salvador de Jujuy.

Briones Gómez, R. 1996. Convertirse en curandero. Legitimidad e identidad social del curandero. En Creer y Curar: La Medicina Popular, editado por J. González Alcantud y S. Rodríguez Becerra, pp. 545-88. Diputación Provincial de Granada, Granada.

Colatarci, M.A. 1999. Reflexiones sobre la iniciación de curanderos en el NOA. Scripta Ethnologica XXI:141-154.
Coreil, J. 1995. Group interview methods in community health research. Medical Anthropology 16:193-210.

Di Lullo, O. 2005 [1947]. El Folklore de Santiago del Estero, Medicina y Alimentación. Fundación Cultural Santiago del Estero, Santiago del Estero.

Disderi, I. 2001. La cura del ojeo. Ritual y terapia en las representaciones de los campesinos del centro-oeste de Santa Fe. Mitológicas XVI:135-151.

Eliade, M. 1968. El Chamanismo y las Técnicas Arcaicas del Éxtasis. Fondo de Cultura Económica, Ciudad de México.

Eliade, M. 1964. Tratado de Historia de las Religiones. Ediciones Era, S.A., Ciudad de México.

Foster, G. 1994. Hipocrates' Latin American Legacy. Humoral Medicine in the New World. Gordon and Breach Science Publishers, New York. 
García, S. 1984. Conocimiento empírico, magia y religión en la medicina popular de los Departamentos de Esquina y Goya (Corrientes). En Cultura Tradicional en el Área del Paraná Medio, editado por F. Bracht, pp. 255-268. Instituto Nacional de Antropología, Buenos Aires.

Gómez García, P. 1996. Teorías étnicas y etnológicas sobre la terapéutica popular. En Creer y Curar: la Medicina Popular, editado por J. González Alcantud y S. Rodríguez Becerra, pp. 223-254. Diputación Provincial de Granada, Granada.

González Alcantud, J. 1996. Alea médica, analogía estructural y apertura utópica. En Creer y Curar: la Medicina Popular, editado por J. González Alcantud y S. Rodríguez Becerra, pp. 589-603. Diputación Provincial de Granada, Granada.

Idoyaga Molina, A. 1997 Ethnomedicine and world-view. A comparative analysis of the rejection and incorporation of the contraceptive methods among argentine women. Anthropology and Medicine 4:145-158.

- - - 1999. Refigurando el shamanismo. Experiencias neoshamánicas en el área metropolitana. En Folklore Latinoamericano, Tomo 1, editado por A. Colatarci, pp. 25-44. INSPF-IUNA, Buenos Aires.

- - - 2000. La calidad de las prestaciones de salud y el punto de vista del usuario en un contexto de medicinas múltiples. Scripta Ethnologica XXII:21-86.

- - - 2001. Lo sagrado en las terapias de las medicinas tradicionales del NOA y Cuyo. Scripta Ethnologica XXIII:9-76.

- - - 2002. Culturas, Enfermedades y Medicinas. Reflexiones sobre la Atención de la Salud en Contextos Interculturales de Argentina. CAEA-CONICET, Buenos Aires.
Kalinsky, B. y W. Arrué 1996. Claves Antropológicas de la Salud. El Conocimiento en una Realidad Intercultural. Miño y Dávila, Buenos Aires.

Krause, C. 2000. Símbolo y procedimiento ritual en dos ceremonias vigentes entre los campesinos de San Juan. Scripta Ethnologica XXII:87-97.

Mariño Ferro, X.R. 1996. La Medicina Popular Interpretada, II. Ediciones Xerais de Galicia S.A, Madrid.

Martínez, G. y A.M. Planchuelo 2003. La medicina tradicional de los criollos campesinos de Paravachasca y Calamuchita (Córdoba Argentina). Scripta Ethnologica XXV:83-116.

Merleau Ponty, M. 1945. La Fenomenología y las Ciencias del Hombre. Biblioteca NOVA de Psicología, Buenos Aires.

Palma, N.H. 1978. La Medicina Popular en el Noroeste Argentino. Ediciones Huemul, Buenos Aires.

Pérez de Nucci, A.1989. Magia y Chamanismo en la Medicina Popular del Noroeste Argentino. Editorial Universitaria de Tucumán, San Miguel de Tucumán.

Ratier, H. 1972. La Medicina Popular. Centro Editor de América Latina, Buenos Aires.

Sturzenegger, O. 1987. Médecine Traditionnelle et Pluralisme Médical dans une Culture Créole du Chaco Argentin. Memoire du DEA, Paris.

Taussig, M. 1987. Shamanism, Colonialism and the Wild Man: A study in terror and healing. The University of Chicago Press, Chicago.

Torres, G. y C. Aprea 1995. Un ejemplo de la vigencia de sistemas médicos paralelos en los estratos marginales del Gran Buenos Aires: La pata de cabra. Kallawaya, nueva serie, 2:87-106.

\section{Notas}

1 Al hablar de medicina tradicional nos referimos a una categoría hueca que se llena a partir del conocimiento etnográfico de distintas localidades o regiones (Idoyaga Molina 2002) y debe ser analizada no en sí misma, sino referida al sistema cultural dentro del cual el grupo utiliza un espectro de representaciones y prácticas provenientes de dicha medicina. En este trabajo, tomaremos la conceptualización que nos brinda Idoyaga Molina (2002), quien las define sobre la base de dos instituciones, los shamanismos y el curanderismo. A su vez este último genera una forma de autotratamiento tradicional que es conocida con el nombre de medicina casera o doméstica.

2 Este grupo en la zona de estudio se autodenominaba Ranqueles.

3 El mal de ojo u ojeo es un desequilibrio social que puede ser especialmente dañino para los niños. Se manifiesta generalmente como un dolor intenso de cabeza o cefalea, lo cual en los niños se deja ver en el llanto constante. Su etiología encuentra más de un origen, uno remite a la intención negativa de algún mayor que con malos sentimientos o "envidia" miran, tocan o piensan en los niños (Bianchetti 1994; Disderi 2001; Idoyaga Molina 2002; Palma 1978; Pérez de Nucci 1989). La otra opción es no intencional y sucede cuando una persona adulta con mucho poder o portadora de una mirada muy fuerte toca o mira al infante que finalmente resulta ojeado. El empacho es un taxón frecuente que puede ser padecido por individuos de todas las edades, pero la gran mayoría de los casos observados son en infantes y chicos de corta edad; sin embargo, también encontramos casos de adolescentes y algunos mayores. Es un malestar estomacal que acarrea algunas veces cefaleas, las que se definen como un síntoma derivado del trastorno intestinal. Su etiología, entre todos los casos recogidos, está asociada a desbalances térmicos y alimenticios, tales como la ingesta de una comidas o bebidas demasiado frías o demasiado calientes, especialmente si el cuerpo no se halla en condición templada, a los excesos de alimentación y/o bebida, a combinaciones de alimentos y bebidas inadecuados -por ejemplo, sandía y vino tinto-, a comidas "pesadas" o "gordas" -carne de cerdo, cordero, chivito, avestruz- y, en general, a las que se consideran de difícil ingestión -chocolate, polenta, huevos fritos. La culebrilla es un taxón que desde la perspectiva de los actores encuentra diferentes etiologías, puede ser el resultado de factores exógenos diversos, tales como el roce de la piel con un reptil minúsculo que recibe el nombre de culebrilla o la introducción en la dermis de los pequeños huevos de una mariposa. Se manifiesta a través de una erupción localizada y agrupada que puede aparecer en cualquier parte del cuerpo. Son vesículas del 
tamaño de la cabeza de un alfiler cuya forma se asemeja a la apariencia de una culebrilla. De acuerdo a los actores sociales, dicha erupción se extiende en sentido ecuatorial hasta formar un círculo, de cerrarse este último el doliente muere indefectiblemente. La pata de cabra, su origen se atribuye a un parásito que se aloja en la espalda a la altura de la cintura baja. Dicho parásito después del transcurso de un tiempo atacaría la médula terminando con la muerte del infante. Se manifiesta a nivel físico con puntos morados alrededor del coxis. Su sintomatología es el llanto constante, ausencia de apetito, fiebre, problemas para caminar y mucho dolor. La brujería o malhecho es un taxón común en el área de estudio, no sólo porque se enmarca de esta manera en el imaginario social, sino porque para los actores es una enfermedad real, diagnosticable y que requiere de una terapéutica especial. Es siempre el resultado de acciones intencionales llevadas a la práctica generalmente por especialistas o por cualquier persona que conozca alguna de las formas (Idoyaga Molina 2002) y están habitualmente dirigidos a personas de la misma comunidad con una intención negativa. La manifestación del daño es variable pues se relaciona con la técnica y los elementos utilizados, la intención de quien lo ejecuta y otros aspectos que hacen que el malhecho pueda producir desde un dolor de cabeza, hasta dificultades para caminar, problemas de presión o cardíacos, pasando por la demencia y manifestaciones sociales como la incapacidad para interactuar con los otros. En este sentido, el daño o brujería es un taxón polimorfo que suele presentarse en desequilibrios orgánicos, emocionales -incluida la locura- y en la imposibilidad de interactuar adecuadamente. Habitualmente conduce a la muerte tal como sucede en otras regiones del país y en el viejo mundo (Arteaga 2006; Bianchetti 1994; Disderi 2001; Idoyaga Molina 1999, 2002; Krause 2000; Mariño Ferro 1996; Palma 1978). Desgarros o aberturas de carnes se denomina a una lesión o tirón muscular. La misma es una rotura parcial o completa de las fibras musculares a causa de un fuerte impacto o excesiva fuerza aplicada a la zona. Además de verse afectadas las fibras musculares, también se puede producir en ligamentos y/o tendones. Su tratamiento se realiza a través de friegas con grasas animales, cura de palabra y la utilización de fármacos de laboratorio.

4 Consideramos el término remedio en un sentido amplio, como superador del significado fármaco-botánico de la expresión "plantas medicinales". De esta manera un vegetal con características terapéuticas no es comparable a la de un medicamento, pudiendo existir plantas sin un registro de principios activos y que pueden ser parte del conocimiento colectivo de propiedades curativas (Martínez y Planchuelo 2003).

5 En lo que hace al concepto de enfermedad seguimos a Idoyaga Molina (1999, 2002), quien, teniendo en cuenta las teorías etiológicas, define la enfermedad como el resultante de diferentes tipos de desequilibrios. Son ellos: (a) Desequilibrios orgánico-emocionales, los males que se manifiestan en nivel físico o emocional y que se deben a causas naturales. (b) Desequilibrios entre las entidades que integran la persona, cuyas dolencias afectan la armonía entre las entidades que integran al individuo, tales como el cuerpo, alma, espíritu. (c) Desequilibrios sociales, se trata de enfermedades que se manifiestan en nivel físico y/o emocional y tienen origen en la acción o el poder de otros individuos, tales como el mal de ojo, la tiricia, la envidia, el daño o malhecho. (d) Desequilibrios espacio-ambientales, se trata de los males causados por espacios de energías negativas, como sucede con algunas concepciones relativas al mal aire. (e) Desequilibrios religioso-rituales, aquellos que se originan en la acción de una deidad, la violación de un tabú, una falla ritual, o una falta moral.

6 El daño existe como taxón de enfermedad en la región del NOA (Bianchetti 1994; Idoyaga Molina 1999, 2000, 2002), en el NEA (Disderi 2001) y en La Pampa (Arteaga 2006), entre otras zonas, haciendo referencia a un desequilibrio vital atribuido a una intencionalidad maligna.

7 Tomamos este concepto en su acepción más amplia del término como algo que manifiesta lo sagrado, incluyendo las deidades pero también las expresiones de poder sin voluntad y sin figura, denominadas por el autor como cratofanías (Eliade 1968:34).

8 De acuerdo a Idoyaga Molina (2001) la cura de palabra, llamada ensalmos en la tradición española, fue traída por los españoles para la época de la Conquista, luego reelaboradas y enriquecidas por los inmigrantes europeos y árabes durante finales del siglo XIX y el XX. Entre los diferentes males que trata se encuentran casi todos los desequilibrios orgánicos, aunque existen oraciones para la cura de enfermedades enmarcadas dentro de los desequilibrios entre las entidades que conforman la persona como el susto y desequilibrios sociales, como el ojeo y el daño. Es también común que se utilice esta forma para la cura de animales. Las fórmulas para curar son secretas y sólo podrán usarlas al momento de la terapia, y las oraciones invocan a la santísima Trinidad y a las figuras del catolicismo, como es el caso de la virgen $\mathrm{y}$ diversos santos.

9 La iniciación a través de la superación de una experiencia crítica como una enfermedad o un accidente es un hecho repetido en los curanderismos de Argentina (Colatarci 1999; Idoyaga Molina 1997, 1999; Palma 1978) y The Last Templar The Last Templar de España (Briones Gómez 1996; González Alcantud 1996; Gómez Becerra 1996). Por otra parte, es también un hecho común dentro de las posibilidades de iniciación descritas por Eliade (1968) en relación con los shamanismos paleo-siberianos. Asimismo aparecen también en los shamanismos Mataco Wichí del gran Chaco en Argentina.

10 Cuando nos referimos a ningún tipo de aviso previo queremos decir que la actora empezó a ser solicitada en prácticas curativas sin ella comentar de su nueva capacidad. Después su eficacia atrajo a nuevos dolientes para su tratamiento. 
